\title{
FUNCTION OF PUMILIO GENES IN HUMAN EMBRYONIC STEM CELLS AND THEIR EFFECT IN STEMNESS AND CARDIOMYOGENESIS
}

Isabelle Leticia Zaboroski Silva ${ }^{1 \#}$, Anny Waloski Robert ${ }^{\sharp \#}$, Guillermo Cabrera Cabo ${ }^{2}$, Lucia Spangenberg ${ }^{2}$, Marco Augusto Stimamiglio ${ }^{1}$, Bruno Dallagiovanna ${ }^{1}$, Daniela Fiori Gradia ${ }^{3 *}$, Patrícia Shigunov ${ }^{1 *}$

${ }^{1}$ Laboratory of Basic Biology of Stem Cells (LABCET), Instituto Carlos Chagas - FIOCRUZPR, Curitiba, Paraná, 81830-010, Brazil

${ }^{2}$ Bioinformatics Unit, Instituto Pasteur de Montevideo. Mataojo 2020, CP 11400, Montevideo, Uruguay.

${ }^{3}$ Department of Genetics, Federal University of Parana (UFPR), Curitiba 81531-980, Brazil

\# Both authors contributed equally to this work.

*To whom correspondence should be addressed at: Laboratory of Basic Biology of Stem Cells (LABCET), Instituto Carlos Chagas - FIOCRUZ-PR, Curitiba, Paraná, Rua Prof. Algacyr Munhoz Mader, 3775, CIC, 81830-010, Brazil.

Tel.: +55 41 21043231; Email: patricia.shigunov@fiocruz.br, danielagradia@ufpr.br

\section{Abstract}

Posttranscriptional regulation plays a fundamental role in the biology of embryonic stem cells (ESCs). Many studies have demonstrated that multiple mRNAs are coregulated by one or more RNA binding proteins (RBPs) that orchestrate the expression of these molecules. A family of RBPs, known as PUF (Pumilio-FBF), is highly conserved among species and has been associated with the undifferentiated and differentiated states of different cell lines. In humans, two homologs of the PUF family have been found: Pumilio 1 (PUM1) and Pumilio 2 (PUM2). To understand the role of these proteins in human ESCs (hESCs), we first demonstrated the influence of the silencing of PUM1 and PUM2 on pluripotency genes. OCT4 and NANOG mRNA levels decreased significantly with the knockdown of Pumilio, suggesting that PUMILIO proteins play a role in the maintenance of pluripotency in hESCs. Furthermore, we observed that the hESCs silenced for PUM1 and 2 exhibited an improvement in efficiency of in vitro cardiomyogenic differentiation. Using in silico analysis, we identified mRNA targets of PUM1 and PUM2 expressed during cardiomyogenesis. With the reduction of PUM1 and 2 , these target mRNAs would be active and could be involved in the progression of cardiomyogenesis.

Keywords: human embryonic stem cells, Pumilio 1, Pumilio 2, cardiomyogenesis, RNA-binding proteins. 


\section{INTRODUCTION}

Human embryonic stem cells (hESCs) are pluripotent cells derived from the inner cell mass of the blastocyst that have potential for differentiation into three germ layers (1-3). In an undifferentiated state, hESCs are characterized by the expression of stemness factors such as OCT4 (POU5F1), SOX2 and NANOG (4). These three transcription factors, which are positively regulated, are responsible for pluripotency maintenance and contribute to the repression of lineage-specific genes (reviewed by 5). When hESCs are stimulated to initiate the differentiation process, expression of genes associated with pluripotency is negatively regulated and genes associated with the germ layer begin to be positively regulated (6).

A complex network of gene expression underlies the molecular signaling that will give rise to the adult heart. Cardiomyogenic differentiation is a highly regulated process that depends on the fine regulation of gene expression (7). In vitro cardiomyogenic differentiation of hESCs mimics embryonic development and can be used as a model for cardiac development studies per se and as a model for research ranging from tissue electrophysiology to drug screening (reviewed by 8 ).

RNA-binding proteins (RBPs) are proteins that have RNA-binding domains and form ribonucleoprotein complexes in association with RNAs (RNPs). These proteins play a critical role in the posttranscriptional regulation of gene expression. The dynamics and function of these complexes depend on their composition, targets and cofactors (9). The PUF (Pumilio-FBF) family of RBPs is highly conserved among species and is found in plants, insects, nematodes and mammals (10-15). PUF proteins have RNA binding domains known as the Pumilio Homology Domain (PUM-HD). The Pumilio RNA interaction domain is highly conserved (16), comprising eight repeats, each having the ability to bind a single nucleotide of specific recognition motif in the $3^{\prime}$ untranslated region (UTR) of a target mRNA (17). In humans, two homologs of the PUF family are found: PUMILIO 1 (PUM1) and PUMILIO 2 (PUM2) which have 91\% identity at the RNA binding domain (15).

The expression of PUM1 and PUM2 has been observed in hESCs and several human fetal and adult tissues, indicating a possible participation in the maintenance of germ cells $(11,12)$. Furthermore, in mammals, the disruption of PUM proteins promotes defective germline phenotypes $(18,19)$. In rodent, PUM1 facilitates the exit from the primitive state to the differentiated form by accelerating the degradation of some 
important elements in the maintenance of pluripotency, such as Tfcp211, Sox2, Tbx3, and Esrrb (20). Besides that, many of the mRNAs associated with PUM1 belong to a relatively small number of functional groups, suggesting an RNA regulon model (21) in which PUM1 inhibits translation and promotes the degradation of its target mRNAs (22). Pumilio proteins form multiprotein complexes with other regulatory proteins, such as DAZ-Like (DAZL) (11), BOULE (BOL) (23), Staufen (STAU) (24) and Nanos (NOS) $(25,26)$. These complexes also are involved in the maintenance of ESC and in the regulation of the onset of meiosis in various organisms, including humans $(11,27,28)$. PUM2 and NOS interact in a conserved mechanism for the development and maintenance of germ cells (29). Thus, the molecular scenario in which Pumilio proteins and their targets work, may lead to cellular differentiation or the maintenance of a stemness phenotype.

Here, we investigated the role of PUM1 and PUM2 proteins in hESCs during cardiomyogenic differentiation. We report that the silencing of PUM1 and PUM2 reduces the expression of pluripotency genes in hESCs. We found that the silencing of PUM1 and PUM2 positively influences cardiomyogenesis in hESCs. Using in silico analysis, we followed the targets of PUM1 and PUM2 during cardiomyogenesis, clustering the targets with the same behavior during differentiation and traced the gene networks.

\section{METHODS}

\section{Cell culture and cardiac differentiation}

The NKX2-5eGFP/w HES3 hESC line (30) was obtained from Monash University (Victoria, Australia) . The cell cultures were maintained on irradiated mouse embryonic fibroblasts (iMEFs) in hESC medium consisting of Dulbecco's modified Eagle's medium (DMEM)/F12 supplemented with 20\% KnockOut serum replacement, $1 \%$ nonessential amino acids, $1 \% \mathrm{~L}$-glutamine, $1 \%$ penicillin/streptomycin, $0.1 \mathrm{mM} \beta$-mercaptoethanol and $10 \mathrm{ng} / \mathrm{ml}$ human $\beta F G F$. The cells were passaged every 3-4 days by enzymatic dissociation using $0.25 \%$ trypsin/EDTA. Cardiomyogenic differentiation assays were conducted using an embryoid body (EB) protocol adapted from previously described $(31,32)$ or a monolayer protocol previously reported $(33)$.

Regarding EB cardiac differentiation protocol, briefly, $7 \times 10^{5}$ cells/well were plated onto Growth Factor Reduced Matrigel ${ }^{\circledR}$ Matrix (Corning) 6-well coated dishes. 
Then, hESCs were dissociated and cultured on low-attachment plates with supplemented StemPro-34 medium (composed of StemPro ${ }^{\text {TM}}-34$ SFM $\left(\right.$ Gibco $\left.^{\text {TM}}\right)$ supplemented with transferrin, ascorbic acid, penicillin/streptomycin and monothioglycerol) containing BMP4 $(0.5 \mathrm{ng} / \mathrm{mL})$ for 24 hours to form EBs (Day 0, D0). On day 1 (D1), the EBs were incubated with supplemented StemPro-34 added with $\beta F G F(5 \mathrm{ng} / \mathrm{ml})$, activin A (6 $\mathrm{ng} / \mathrm{ml})$ and BMP4 (10 ng/ml) to induce mesoderm differentiation. On day 4 (D4), the EBs were incubated with medium supplemented with XAV939 $(10 \mu \mathrm{M} / \mathrm{ml})$ and VEGF $(10 \mathrm{ng} / \mathrm{ml})$ to induce the cells to become cardiac progenitors. On days 8 and 11, the medium was changed to supplemented StemPro-34 containing only VEGF (10 ng/ml). On the $9^{\text {th }}$ day (D9), we observed the expression of eGFP. On day 15 (D15), we evaluated the efficiency of the protocol with cardiac troponin T (cTnT) staining. During the protocol cultivation period, from D0 to D15, the EBs were maintained in a humidified incubator under hypoxic conditions $\left(5 \% \mathrm{O}_{2}, 5 \% \mathrm{CO}_{2}, 37^{\circ} \mathrm{C}\right)$.

For the monolayer cardiac differentiation protocol, hESC were dissociated from iMEF cultures and $1.5 \times 10^{5}$ cells/well were plated onto Matrige ${ }^{\circledR} \mathrm{hESC}$-qualified Matrix (Corning) 24-well coated dishes. hESC were maintained in hESC medium until reached $90-100 \%$ of confluence to started the protocol. At day 0, RPMI medium supplemented with B27 without insulin (RPMI+B27-insulin) and $12 \mu \mathrm{M}$ of CHIR99021 (Stemgent) were added to the culture. After 24h, medium was changed for RPMI+B27-insulin. At day 3, it was added RPMI+B27-insulin and $10 \mu \mathrm{M}$ of XAV939 (Sigma) to the monolayer cultures, which were maintained until day 5, when medium was exchange for the RPMI+B27-insulin. From the $7^{\text {th }}$, the cultures were maintained with RPMI supplemented with B27 complete, with medium exchange every 3 days until the $15^{\text {th }}$. At final day (day 15), cells were fixed with paraformaldehyde $4 \%$ and stained for cTnT using a previously described immunofluorescence protocol (7). Analysis was performed on Operetta CLS High-Content Analysis System and Harmony software (PerkinElmer) (Supplementary Figure S1A).

\section{Lentiviral vector production and transduction}

HEK293FT cells were cultured in Petri dishes containing DMEM supplemented with $10 \%$ fetal bovine serum, $1 \%$ L-glutamine and $1 \%$ penicillin and streptomycin for 24 hours. MISSION Lentiviral Mix and p-KLO1 containing short hairpin RNA (shRNA) targeting PUM1 (shPUM1), PUM2 (shPUM2) or a Scrambled sequence (shSc) 
139 (34) were added to the cell culture in OptiMEM containing Lipofectamine 2000 for 4 140 hours. Then, the medium was replaced with supplemented DMEM, as described above. 141 After 48 and 72 hours, the medium was collected and centrifuged twice at $141000 \times \mathrm{g}$. 142 The cell pellet was resuspended in $1 \mathrm{X}$ PBS and stored at $-80^{\circ} \mathrm{C}$.

$143 \quad$ For transduction, hESCs were cultured on 6-well plates and different dilutions of 144 the lentiviruses were tested (Supplementary Figure 2), and the dilution was defined as $14510^{-3}$ for all experiments. After the transduction, the medium was replaced and hESCs 146 were cultured for 24 hours to induce cardiac differentiation.

\section{RNA extraction and quantitative RT-qPCR}

RNA was extracted using the RNeasy Kit (Qiagen), and the cDNA reaction was performed using the Improm II Kit (Promega) according to the manufacturer's instructions. Samples were obtained from three replicates for undifferentiated cells and from three independent cardiac differentiations. Amplifications were carried out in a final volume of $10 \mu \mathrm{l}$ containing SYBR Select master mix (Applied Biosystems), $100 \mathrm{ng}$ cDNA template, and 5-10 pmol primers. The RT-qPCR conditions followed the manufacture's recommendations (Applied Biosystems), using the LightCycler system (Roche). The melting curves were acquired after RT-qPCR to confirm the specificity of the amplified products (Supplementary Table S1). We generated standard curves for each gene, including the GAPDH (housekeeping) gene. Amplifications were performed in triplicate. $\mathrm{Cq}$ results for each gene were normalized based on GAPDH expression and the analysis of relative expression were performed.

\section{Flow cytometry}

During cardiac differentiation, the cells were immunophenotyped by flow cytometry to confirm differentiation stages. The EBs were dissociated with $0.25 \%$ trypsin/EDTA (5 min) and resuspended in PBS/0.5\% BSA. On day 3, the cells were incubated with anti-CD56 (BD) antibody $(1: 12.5)$ for 20 min at $4{ }^{\circ} \mathrm{C}$. On day 9 , the cells were only dissociated for eGFP detection. On day 15, for cTnT staining, the EBs were incubated with trypsin/0.25\% EDTA for $20 \mathrm{~min}$, followed by inactivation with DMEM supplemented with 50\% SFB and DNase I (20-30 U/ml). After dissociation, the cells were fixed with $4 \%$ formaldehyde $(20 \mathrm{~min}$ ), followed by permeabilization with $0.5 \%$ Triton X-100 (25 min). Then, the cells were incubated with an anti-cTnT primary 
172 antibody (Thermo Fisher Scientific) (1:100) for $30 \mathrm{~min}$ at room temperature, followed

173 by incubation with Alexa Fluor 633 secondary antibody (1:1000) (30 min). The cells

174

175

176

177 were analyzed on a FACSCanto II (BD) flow cytometer. Data analysis was performed using FlowJo software (v.10).

\section{Immunofluorescence}

The immunofluorescence protocol followed as previously described (7). Briefly, monolayer cultures we fixed with paraformaldehyde 4\%, rinsed with PBS, followed by incubation with blocking buffer (PSA/BSA 5\%) for $60 \mathrm{~min}$. Then, cells were incubated with primary antibodies for Pumilio 1 (1:300, Bethyl laboratories Inc.), Pumilio 2 (1:70, Bethyl laboratories Inc.) or OCT4 (1:100, Abcam), diluted in blocking buffer, for 60 min, at $30^{\circ} \mathrm{C}$. After three PBS washes, cells were incubated with Alexa Fluor ${ }^{\circledR} 488$ antigoat or anti-rabbit secondary antibody, for $60 \mathrm{~min}$, at $30^{\circ} \mathrm{C}$. DAPI were staining for 10 min, followed by three PBS washes. Analysis was performed on Operetta CLS HighContent Analysis System and Harmony software (PerkinElmer) (Supplementary Figure S1B).

\section{Analysis of mRNA targets of PUM1 and PUM2}

To analyze the mRNA targets of PUM1 and PUM2 proteins through cardiac differentiation, we used the RNA-seq data from mRNAs associated with polysomes obtained during in vitro cardiomyogenesis previously published (32). Initially we defined a set of 1,809 target genes for human Pumilios from published (22). Differential expression analysis was done using the Bioconductor $\mathrm{R}$ package edgeR $(35,36)$. Comparisons were performed for polysome-bound RNA fractions - each sample against the preceding time-point: Day 0 vs Day 1 and Day 0 vs Day4. For these analyses, we retained only those genes with at least one count per million in at least three samples. Based on initial results of DEGs we used a stringent analysis using a p-value threshold of 0.05 and a $\log 2$ Fold change $(\log \mathrm{FC})$. Genes with a $\log \mathrm{FC}>1$ were considered upregulated, and genes with a $\operatorname{logFC}<-1$ were considered downregulated. An enrichment analysis of this set of genes was performed using g:Profiler (37) (http://biit.cs.ut.ee/gprofiler/) and REVIGO (38) (http://revigo.irb.hr/) consortium database. Complement analysis was performed with STRING CONSORTIUM 2019 (39) (https://string-db.org). 


\section{Statistical analysis}

Statistical analysis was performed using GraphPad Prism 7 software. The data sets are expressed as the means \pm standard deviation. According to data sets were used unpaired Student's t-test or one-way ANOVA followed by Tukey post hoc test. Differences with $\mathrm{p}<0.05$ were considered statistically significant.

\section{RESULTS}

Differences in PUM1 and PUM2 expression comparing total and polysomal RNA

To evaluate the expression levels of PUM1 and PUM2 throughout cardiomyogenesis, hESCs were submitted to an in vitro cardiac differentiation protocol and its progression was verified by flow cytometry (Figure 1A). On D3, about $25 \%$ of the cell population was $\mathrm{CD}^{+} 6^{+}$(mesoderm marker) indicating an advanced process of mesodermal differentiation (Figure 1B). On D9, the expression of eGFP, under the control of the NKX2.5 promoter (cardiac progenitor marker), was evaluated and approximately $20 \%$ of the cell population expressed the marker, confirming the cardiac commitment of this population. On the $15^{\text {th }}$ day, the expression of the cardiomyocyte marker cTnT was assessed, showing positive labeling for approximately $20 \%$ of the cell population (Figure 1B).

Total RNA was extracted from 5 time-points during cardiac differentiation (D0, D1, D4, D9, D15) and RT-qPCR was performed. The expression analysis of PUM1 and PUM2 mRNAs showed a slight increase throughout the differentiation process (Figure 1C). In previous work, we analyzed the expression of polysome-associated mRNAs along cardiomiogenesis (32). Interestingly, when we analyzed the association of PUM mRNAs with polysomes along cardiomyogenesis, based on the fold change of Reads Per Kilobase Million (RPKM) values comparing each time-point in relation to D0, we noticed no significant changes (Figure 1D).

Individual and combined knockdown of $P U M 1$ and $P U M 2$ affects the expression of $O C T 4$ and $N A N O G$ in hESCs.

To understand the role of PUM1 and PUM2 in hESC maintenance or during cardiomyogenic differentiation, we silenced their expression using short hairpin RNAs. 
scrambled control from previously published plasmids (34) (Supplementary Figure S2). After $24 \mathrm{~h}$ of hESCs transduction, the cells showed no evident morphological changes (Figure 2A). Knockdown of PUM1 and PUM2 was confirmed by RT-qPCR, which showed a significant reduction of PUM1 and PUM2 mRNA levels under silencing conditions compared to the control in single- and double-silenced cells (Figures 2B-C). shPUM1 also interfered in reducing the expression levels of PUM2 mRNA and vice versa, suggesting that the shRNAs presented a low specificity or the PUM proteins could be regulating each other. Double-silencing showed better efficiency in silencing both Pumilio genes: $92 \%$ for $P U M 1$ and $90 \%$ for PUM2.

Then we observed whether the silencing of PUM1 and PUM2 altered the mRNA levels of some pluripotency transcription factors, as OCT4 and NANOG. Interestingly, the mRNA levels were significantly reduced for both genes in cells with PUM1 or PUM2 silencing and double silencing relative to the control (Figures 2D-E). Considering the efficacy of shPUM1-2 in reducing Pumilios and OCT4 mRNA expression, we quantified the protein content. Quantification of cytoplasmatic PUMI and PUM2 and nuclear OCT4 staining intensity comparing hESC cultures transduced with shPUM1-2 with those transduced with shSc (Figure 2F-H) indicated a decrease in protein quantity. These results suggest that PUM1 and PUM2 could regulate positively of OCT4 and NANOG mRNAs.

\section{Knockdown of PUM1 and PUM2 in hESCs affects cardiomyogenesis.}

Considering the effects of Pumilio silencing in pluripotency factors, we also evaluated their influence in cardiomyogenesis. After $48 \mathrm{~h}$ of hESC transduction, cardiac differentiation was induced. The transduction did affect neither the morphology of cells nor the formation of EBs (D1 and D4) (Figure 3A). From the $9^{\text {th }}$ day, silenced EBs, visually, presented some morphological differences with respect to their size compared to the control cells (Figure 3A), though this difference was not statistically significant (Figure 3B). In all conditions, the EBs contract spontaneously (Supplementary Video $1)$.

Analysis comparing shSc with shPUM knockdown conditions (shPUM1, shPUM2 and shPUM1-2) showed that cells transduced with shPUM2 and shPUM1-2 increase the number of eGFP+ cells at D9 compared to cells transduced with shSc, but not at D15 (Figure 3C). Regarding the efficiency of differentiation, on D15, the 
271 percentage of cTnT + cells not changed between the different treatments (Figure 3C).

272 These results demonstrated that when PUM was silenced, hESCs followed EBs cardiac differentiation efficiently, with no statistically significant changes.

We performed a monolayer cardiomyogenic differentiation protocol, as previously described (33) (Figure 4A). In this protocol we transfected hESCs with shSc or shPUM1-2 and analyzed the efficiency of cardiac differentiation through eGFP/Nkx2.5 expression and cTnT immunostaining at day 15. During the differentiation process, it was observed that PUM1-2 silenced hESC had more beating areas in the monolayer culture than the shSc transduced cells, which were maintained until the end of the protocol (Supplementary Video 2). The beating frequency of shPUM1-2 transduced cells is on average $7( \pm 2.9)$ contractions in 10 seconds while shSc transduced cells showed an average of $3( \pm 1.3)$ contractions in 10 seconds $(n=3, p<0,01)$ (Supplementary Video 2). Thus, the silencing of PUM1-2 increases the frequency of contractions relative to the shSc control. Also, the $\mathrm{eGFP}^{+}$and $\mathrm{cTnT}^{+}$stained areas, at the end of protocol, were significantly higher in shPUM1-2 treated cells, with no changes in cell number (DAPI ${ }^{+}$area) (Figure 4B-D). Our data suggest that PUM1 and PUM2 could play a negative role in the control of cardiac lineage commitment.

mRNA targets of PUM1 and PUM2 involved in cardiac development are associated with polysomes during cardiomyogenesis

Our results suggest that PUM1 and PUM2 may play a role in the cardiac differentiation process. Thus, we investigated the expression profile of the targets mRNA of PUM1 and PUM2 during cardiomyogenesis of hESCs using previously published data (32). Briefly, mRNA population associated with polysomes in five timepoints (days 0, 1, 4, 9 and 15) during the in vitro cardiac differentiation of hESCs were isolated and sequenced (32). Using these data, we selected mRNA targets of PUM1 and PUM2 and we evaluated the genes that increase after 1 and 4 days of cardiomyogeneses (Supplementary Table S2).

Gene ontology (GO) analysis for each set of mRNAs targets of PUM1 and (http://biit.cs.ut.ee/gprofiler/). The complete lists of GO analysis concerning cellular components, biological processes, molecular functions and signaling pathways could be found in the Supplementary Tables S3. In order to aid visualization of the GO terms 
304 found for biological processes we use the REVIGO (38) (http://revigo.irb.hr/). The mRNAs targets of PUM1 and PUM2 were related with biological process as stem cell development, mesenchymal cell differentiation, head development and others (Figures 5A). Networks analysis of these same genes presents elements involved with circulatory system development, among these genes are: EPOR, WNT5A, EFNA1, RHOB, HOXB3, BMP2, SMAD7, FN1, ADAMTS6, COL15A1 and COL4A2. These findings may underlie the fact that PUM1 and PUM2 silencing has favored cardiomyogenesis, since these mRNAs associated with circulatory system development may be more activated.

\section{DISCUSSION}

The PUF family of RNA-binding proteins is involved in these events in higher eukaryotes (40) . In humans, PUM1 and PUM2 are coexpressed in several tissues, and almost $90 \%$ of the PUM2 target genes are also targets of PUM1, showing that human PUM proteins have many similarities in substrate specificity and eventually act redundantly on common targets $(22,41)$. In this context, hESCs may help to clarify the role of these proteins, either in pluripotency maintenance or during a specific lineage differentiation.

In this work, when monitoring PUM1 and PUM2 expression during the cardiomyogenic differentiation of hESC, we observed that PUMI expression increased over time. Distinct Pumilio expression profiles were observed during mouse hematopoietic (12) and human adipogenic differentiation (42). However, when we analyzed PUM1 and PUM2 expression profile during cardiomyogenesis (32) we observed that both mRNAs appear associated to polysomes in a constant pattern throughout the whole process, indicating that the maintenance of PUM1 and PUM2 mRNA levels is important for cell function.

Therefore, we investigated the impact of Pumilio proteins on stemness and cardiomyogenesis by knocking down the two human paralogs, individually and in combination. As discussed previously, PUM1 and PUM2 share most of their mRNA targets $(22,41)$, and a compensatory regulation mechanism has been observed when one of these genes is silenced by increasing the expression of the other (43). We evaluated the levels of $P U M 1$ and PUM2 mRNAs after silencing these genes individually, and we 
337 did not observe this compensation, at least at the mRNA level. We hypothesized that due to their high similarity, the shRNA used to knockdown one transcript impacted the stability of the other transcript, at least in this cell type.

We observed that the PUM1 and PUM2 silencing altered the mRNA levels of two genes associated with the pluripotency phenotype, OCT4 and NANOG. In both cases, the expression was significantly reduced in silenced cells in relation to the control, suggesting that PUM proteins may be involved in the maintenance of hESC pluripotency. These results corroborate with other studies that strongly suggest that PUF proteins may mediate a widespread and ancient mechanism for repressing the differentiation and maintaining the self-renewal of stem cells $(10,15,44-47)$.

As Pumilio proteins seem to regulate the stemness phenotype, we examined the effect of their silencing on hESC induced to two different protocols of in vitro cardiomyogenesis. In EBs protocol, hESC silenced for PUM1 and/or PUM2 showed an increase in the percentage of $\mathrm{cTnT}^{+}$cells, despite it was not statistically significant. However, it is important to point out that the percentage of positive cells during EBs cardiac differentiation protocol may even be higher, since the dissociation of EBs containing organized cell-cell junctions becomes difficult, which may impair quantification by flow cytometry. Moreover, using the monolayer protocol we observed that PUM1-2 silenced hESC exhibited a larger cTnT staining area than control cells. These changes indicated that the reduction of PUM1 and PUM2, even over a short period, can increase the number of cardiomyocytes generated from in vitro cardiac differentiation protocols. The mechanism behind these effect needs to be investigated but could be related to the decrease of pluripotent markers.

Because stemness and differentiation are both subjected to Pumilio regulation, based on the data previously generated from mRNA sequencing from free and polysomal fractions of hESC induced to cardiomyogenesis (32), we performed a bioinformatic analysis of the expression profiles of the PUM targets. We identified two clusters of genes consistently increasing their representation during differentiation. GO analysis revealed that genes related to nervous system development were present in the free fraction, while in the polysomal fractions we identified more genes related to mesodermal and cardiac development. This evidence suggested that mRNAs associated with other germ layers were enriched in the free fraction and, possibly, are being switched off during the cardiac differentiation process. On the other hand, genes related 
370 to cardiomyogenic lineage are associated with polysomes, suggesting, as expected, that

371 these genes are expressed and translated. Besides that, among Pumilios targets mRNAs

372 we identified many transcription factors, which are key components of differentiation 373 processes. These results suggest that PUM1 and PUM2 could be regulators of 374 transcription factors expression during cardiomyogenesis.

$375 \quad$ Here we demonstrated that PUM1 and PUM2 are expressed throughout of hESC 376 in vitro cardiomyogenesis differentiation. Moreover, when both genes were knocked 377 down, we observed a reduction in pluripotency transcription factors expression and an 378 increase in cardiac differentiation efficiency. Through analysis of previous published 379 RNAseq data, we could identify many PUM targets mRNA modulated during 380 cardiomyogenesis. Despite that, the mechanism of these proteins in cardiac lineage 381 commitment still needs to be elucidated. 


\section{Ethics approval and consent to participate}

388 Not applicable.

\section{Competing interests}

391 The authors report there are no potential conflicts of interest or financial interests.

\section{Funding}

This study was supported by FIOCRUZ.

\section{Authors' contributions}

397 ILZS and AWR carried out the molecular studies. GCC and LS carried out the 398 bioinformatics analysis. AWR and MS carried out the cytometry analysis. BD review the manuscript. DFG and PS wrote the manuscript. All authors read and approved the final manuscript.

401

\section{Acknowledgments}

The authors thank the Program for Technological Development in Tools for HealthPDTIS/FIOCRUZ for the use of its facilities. We also thank Wagner Nagib de Souza Birbeire for editing the videos.

\section{REFERENCES}

1. Bradley A. Embryonic Stem Cells: proliferation and differentiation. Curr Opin Cell Biol. 1990;2:1013-7.

410 2. Fuchs E, Segre J a. Stem cells: a new lease on life. Cell. 2000;100(1):143-55.

411 3. Thomson JA, Itskovitz-eldor J, Shapiro SS, Waknitz MA, Swiergiel JJ, Marshall VS, et

412 al. Embryonic Stem Cell Lines Derived from Human Blastocysts. Science (80- ).

$413 \quad 1998 ; 282: 1145-8$.

414 4. Carpenter MK, Rosler E, Rao MS. Characterization and Differentiation of Human $415 \quad$ Embryonic Stem Cells. Cloning Stem Cells. 2003;5(1):79-88.

416 5. Young RA. Control of the embryonic stem cell state. Cell [Internet]. 2011;144(6):940-

417 54. Available from: http://dx.doi.org/10.1016/j.cell.2011.01.032

418 6. Stumpf PS, Smith RCG, Lenz M, Schuppert A, Babtie A, Chan TE, et al. Stem cell 
differentiation is a stochastic process with memory. bioRxiv. 2017;1-26.

7. Pereira IT, Spangenberg L, Robert AW, Amorín R, Stimamiglio MA, Naya H, et al. Cardiomyogenic differentiation is fine-tuned by differential mRNA association with polysomes. BMC Genomics. 2019;20(1):219.

8. Mummery CL, Zhang J, Ng ES, Elliott DA, Elefanty AG, Kamp TJ. Differentiation of Human Embryonic Stem Cells and Induced Pluripotent Stem Cells to Cardiomyocytes: A Methods Overview. Circ Reasearch. 2012;344-59.

10. Parisi M, Lin H. The Drosophila pumilio Gene Encodes Two Functional Protein Isoforms That Play Multiple Roles in Germline Development, Gonadogenesis ,. Genetics. 1999;153:235-50.

11. Moore FL, Jaruzelska J, Fox MS, Urano J, Firpo MT, Turek PJ, et al. Human Pumilio-2

12. Spassov DS, Jurecic R. Mouse Pum1 and Pum2 genes, members of the Pumilio family

13. Abbasi N, Park Y-I, Choi S-B. Pumilio Puf domain RNA-binding proteins in

16. Zamore PD, Williamson JR, Lehmann R. The Pumilio protein binds RNA through a

17. Edwards TA, Pyle SE, Wharton RP, Aggarwal AK. Structure of pumilio reveals 
[Internet]. 2016;94(6):134-134. Available from: https://academic.oup.com/biolreprod/article-lookup/doi/10.1095/biolreprod.115.137497

19. Xu EY, Chang R, Salmon NA, Pera RAR. A Gene Trap Mutation of a Murine Homolog of the Drosophila Stem Cell Factor Pumilio Results in Smaller Testes But Does Not Affect Litter Size or Fertility. Mol Reprod Dev. 2007;74:912-21.

20. Leeb M, Dietmann S, Paramor M, Niwa H, Smith A. Genetic exploration of the exit from self-renewal using haploid embryonic stem cells. Cell Stem Cell. 2014;14(3):385-93.

21. Keene JD. RNA regulons: coordination of post-transcriptional events. Nat Rev Genet. 2007;8(7):533-43.

22. Galgano A, Forrer M, Jaskiewicz L, Kanitz A, Zavolan M, Gerber AP. Comparative analysis of mRNA targets for human PUF-family proteins suggests extensive interaction with the miRNA regulatory system. PLoS One. 2008;3(9).

23. Urano J, Fox MS, Reijo Pera RA. Interaction of the conserved meiotic regulators, BOULE (BOL) and PUMILIO-2 (PUM2). Mol Reprod Dev. 2005;71(3):290-8.

24. Dubnau J, Chiang AS, Grady L, Barditch J, Gossweiler S, McNeil J, et al. The staufen/pumilio pathway is involved in drosophila long-term memory. Curr Biol. 2003;13(4):286-96.

25. Wreden C, Verrotti AC, Schisa JA, Lieberfarb ME, Strickland S. Nanos and pumilio establish embryonic polarity in Drosophila by promoting posterior deadenylation of hunchback mRNA. Development. 1997;124:3015-23.

26. Forbes A, Lehmann R. Nanos and Pumilio have critical roles in the development and function of Drosophila germline stem cells. Development. 1998;125(4):679-90.

27. Fox M, Urano J, Pera RAR. Identification and characterization of RNA sequences to which human PUMILIO-2 ( PUM2 ) and deleted in Azoospermia-like ( DAZL ) bind. Genomics. 2005;85:92-105.

28. Xu EY, Moore FL, Pera RA. A gene family required for human germ cell development evolved from an ancient meiotic gene conserved in metazoans. Proc Natl Acad Sci U S A [Internet]. 2001;98(13):7414-9. Available from: http://www.pubmedcentral.nih.gov/articlerender.fcgi?artid=34683\&tool=pmcentrez\&ren dertype $=$ abstract

29. Jaruzelska J, Kotecki M, Kusz K, Spik A, Firpo M, Reijo Pera R a. Conservation of a Pumilio-Nanos complex from Drosophila germ plasm to human germ cells. Dev Genes Evol. 2003;213(3):120-6.

30. Elliott DA, Braam SR, Koutsis K, Ng ES, Jenny R, Lagerqvist EL, et al. NKX2$5 \mathrm{eGFP} / \mathrm{w}$ hESCs for isolation of human cardiac progenitors and cardiomyocytes. Nat Methods. 2011;8(12):1037-43. 
31. Kattman SJ, Witty AD, Gagliardi M, Dubois NC, Niapour M, Hotta A, et al. Stagespecific optimization of activin/nodal and BMP signaling promotes cardiac differentiation of mouse and human pluripotent stem cell lines. Cell Stem Cell [Internet]. 2011;8(2):228-40. Available from: http://dx.doi.org/10.1016/j.stem.2010.12.008

32. Pereira IT, Spangenberg L, Robert AW, Amorín R, Stimamiglio MA, Naya H, et al. Polysome profiling followed by RNA-seq of cardiac differentiation stages in hESCs. Nat Publ Gr [Internet]. 2018;5:1-11. Available from: http://dx.doi.org/10.1038/sdata.2018.287

33. Lian X, Zhang J, Azarin SM, Zhu K, Hazeltine LB, Bao X, et al. Directed cardiomyocyte differentiation from human pluripotent stem cells by modulating Wnt/B-catenin signaling under fully defined conditions. Nat Protoc. 2012;8(1):162-75.

34. Miles WO, Tschöp K, Herr A, Ji JY, Dyson NJ. Pumilio facilitates miRNA regulation of the E2F3 oncogene. Genes Dev. 2012;26(4):356-68.

35. Huber W, Carey VJ, Gentleman R, Anders S, Carlson M, Carvalho BS, et al. Orchestrating high-throughput genomic analysis with Bioconductor. Nat Methods. $2015 ; 12: 115-21$.

36. Gentleman R, Carey VJ, Bates DM, Bolstad B, Dettling M, Dudoit S, et al. Bioconductor: open software development for computacional biology and bioinformatics. Genome Biol. 2004;5(10):R80.

37. Reimand J, Arak T, Adler P, Kolberg L, Reisberg S, Peterson H, et al. g:Profiler-a web server for functional interpretation of gene lists (2016 update). Nucleic Acids Res. 2016;44(W1):W83-9.

38. Supek F, Bošnjak M, Škunca N, Šmuc T. REVIGO summarizes and visualizes long lists of gene ontology terms. PLoS One. 2011;6(7):e21800.

39. Szklarczyk D, Gable A, Lyon D, Junge A, Wyder S, Huerta-Cepas J, et al. STRING V11: protein-protein association networks with increased coverage, supporting functional discovery in genome-wide experimental datasets. Nucleic Acids Res. 2019;47(D8):D607-13.

40. Quenault T, Lithgow T, Traven A. PUF proteins: Repression, activation and mRNA localization. Trends Cell Biol [Internet]. 2011;21(2):104-12. Available from: http://dx.doi.org/10.1016/j.tcb.2010.09.013

41. Bohn JA, Etten JL Van, Schagat TL, Bowman BM, Mceachin C, Freddolino PL, et al. Identification of diverse target RNAs that are functionally regulated by human Pumilio proteins. Nucleic Acids Res. 2017;(November):1-25.

42. Shigunov P, Sotelo-Silveira J, Kuligovski C, Aguiar AM, Rebelatto CK, Moutinho A, et al. PUMILIO-2 Is Involved in the Positive Regulation of Cellular Proliferation ih Human 
Adipose-Derived Stem Cells. Stem Cells Dev. 2012;21(2):217-27.

43. Kedde M, van Kouwenhove M, Zwart W, Jude Vrielink J, Elkon R, Agami R. A Pumilio-induced RNA structure switch in p27-3' UTR controls miR-221 and miR-222 accessibility. Nat Cell Biol. 2010;12(10):1014-20.

44. Wickens M, Bernstein DS, Kimble J, Parker R. A PUF family portrait: 3'UTR regulation as a way of life. Trends Genet. 2002;18(3):150-7.

45. Crittenden SL, Bernstein DS, Bachorik JL, Thompson BE, Gallegos M, Petcherski AG, et al. A conserved RNA-binding protein controls germline stem cells in Caenorhabditis

46. Ariz M, Mainpal R, Subramaniam K. C. elegans RNA-binding proteins PUF-8 and MEX-3 function redundantly to promote germline stem cell mitosis. Dev Biol. 2009;326(2):295-304.

47. Salvetti A, Rossi L, Lena A, Batistoni R, Deri P, Rainaldi G, et al. DjPum, a homologue

FIGURE LEGENDS

Figure 1. PUM1 and PUM2 expression profile during cardiomyogenesis of hESC. (A) of Drosophila Pumilio, is essential to planarian stem cell maintenance. Development. 2005;132(8):1863-74. percentage of positive cells for the markers CD56, eGFP/Nkx2.5 and cTnT in different timepoints of in vitro cardiac differentiation. (C) Relative expression of PUM1 and PUM2 during expression of RPKM values of PUM1 and PUM2 mRNAs associated with polysomes during in vitro cardiomyogenesis, in relation to day 0 (Data extracted from previously published results by 31). ${ }^{*} \mathrm{p}<0.05$ in relation to $\mathrm{D} 1$.

Figure 2. Knockdown of PUM1 and PUM2 affects the expression of OCT4 and NANOG. (A) Transduction scheme of hESC before EBs cardiac differentiation. (B-E) Analysis of the transduction with lentiviral vectors containing shSc, shPUM1, shPUM2 and shPUM1-2. Representative images of immunostaining and its respective intensity quantification of PUM1 (F), PUM2 (G) and OCT4 (H) after $24 \mathrm{~h}$ of transduction with lentiviral vectors containing shSc 
562 Figure 3. Effect of knockdown of PUM1 and PUM2 during EBs cardiac differentiation. (A)

563 Morphology of EBs at days 1 (D1), 4 (D4), 9 (D9) and 15 (D15) of cardiomyogenesis

564 differentiation of hESC previously transduced with shSc and shPUM1-2. Scale bars: $100 \mu \mathrm{m}$.

565 (B) Area of EBs after 9 days of in vitro cardiac differentiation. (C) Percentage of positive cells

566 for CD56, eGFP/Nkx2.5 and cTnT during cardiomyogenesis of the cells silenced for scramble,

$567 \quad$ PUM1, PUM2 and PUM1-2. * $\mathrm{p}<0.05$

568

569 Figure 4. Effect of knockdown of PUM1 and PUM2 during monolayer cardiac

570 differentiation. (A) Scheme of transduction and monolayer cardiomyogenic differentiation

571 protocol of hESC. (B) Representative images of immunofluorescence of transduced hESC

572 induced with a monolayer cardiac differentiation protocol, after 15 days. Scale bars: $500 \mu \mathrm{m}$.

573 Quantification of $\mathrm{DAPI}^{+}(\mathrm{C})$, eGFP/Nkx2.5 $(\mathrm{D})$ and $\mathrm{cTnT}^{+}(\mathrm{E})$ staining areas after 15 days of

574 monolayer cardiac differentiation. * $\mathrm{p}<0.05$

575

576 Figure 5. mRNA targets of PUM1 and PUM2 upregulated in the polysome during

577 cardiomyogenesis. (A) Gene ontology analysis of mRNAs targets of PUM1 and PUM2

578 upregulated in 4 days of cardiomyogenesis. The figure shows a REVIGO scatterplot of the

579 representative clusters of GO terms obtained with g:Profiler. In the two-dimensional space of the

580 graph, the $\log 10 \mathrm{p}$-value of each GO after REVIGO analyses is plotted on the $\mathrm{x}$-axis, while the

581 terms are scattered based on log size on the $y$-axis. Bubble color indicates the provided $\mathrm{p}$-value

582 (legend). (B) Networks analysis of mRNAs targets of PUM1 and PUM2 upregulated in 4 days

583 of cardiomyogenesis. Functional enrichments in circulatory system development (FDR 1.99e-

584 05) were represented in red. Interaction Score: Highest confidence (0.900), STRING. 
$A$

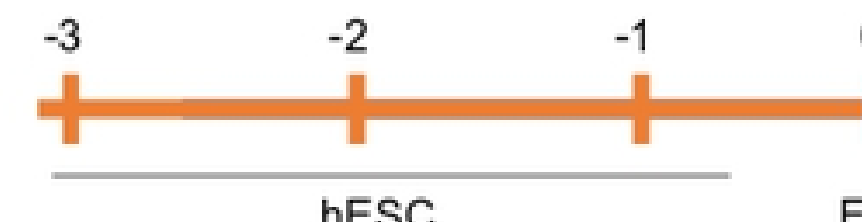

(monolayer culture)

Embryoid bodies (EBs) formation
Cardiac progenitors (eGFP/Nkx2.5+ cells)

\section{Cardiac mesoderm}

C

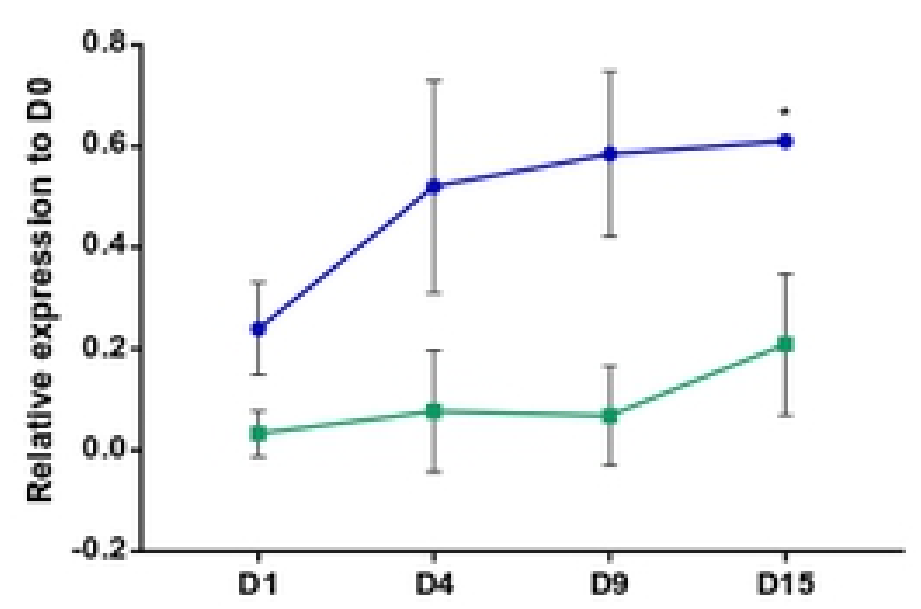

D

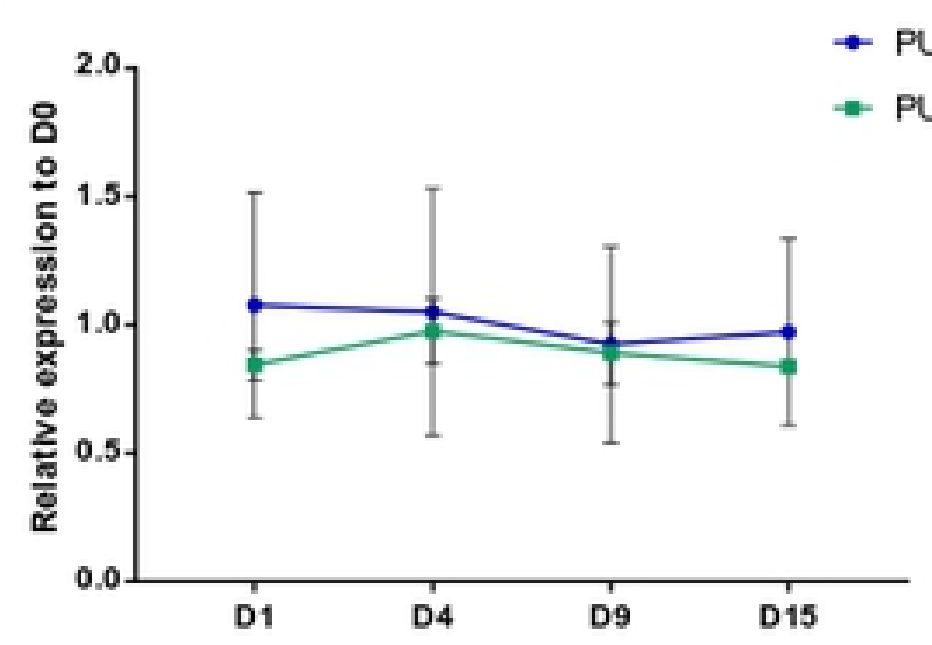

Figure 1 
A

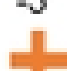
hESC seeding (monolayer)

Lentiviral hESC transduction recovery

EB cardiac

differentiation

B

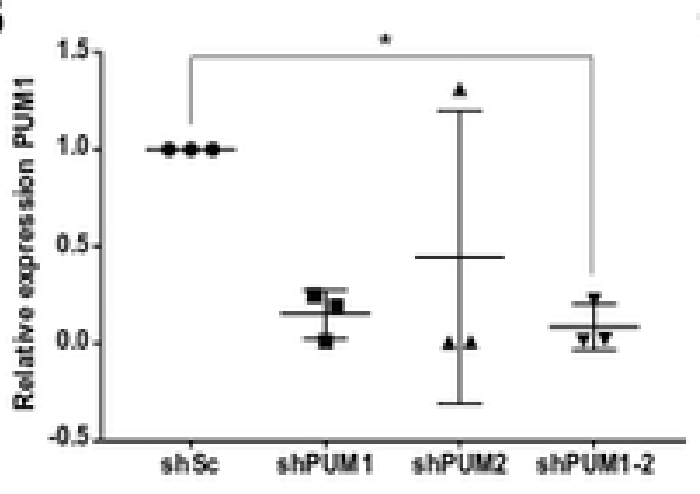

C

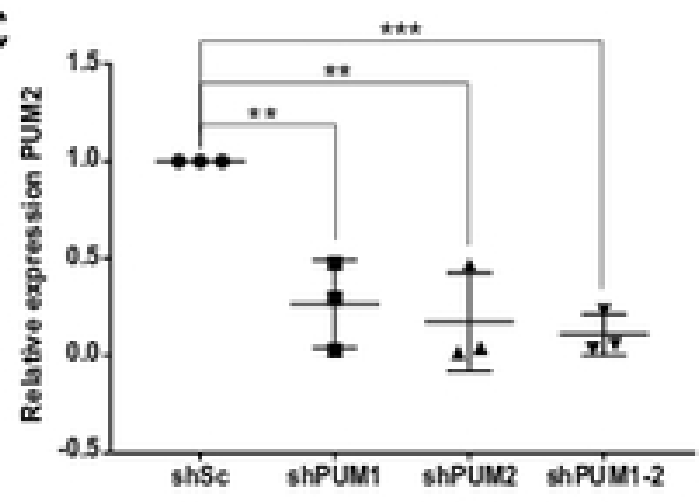

D
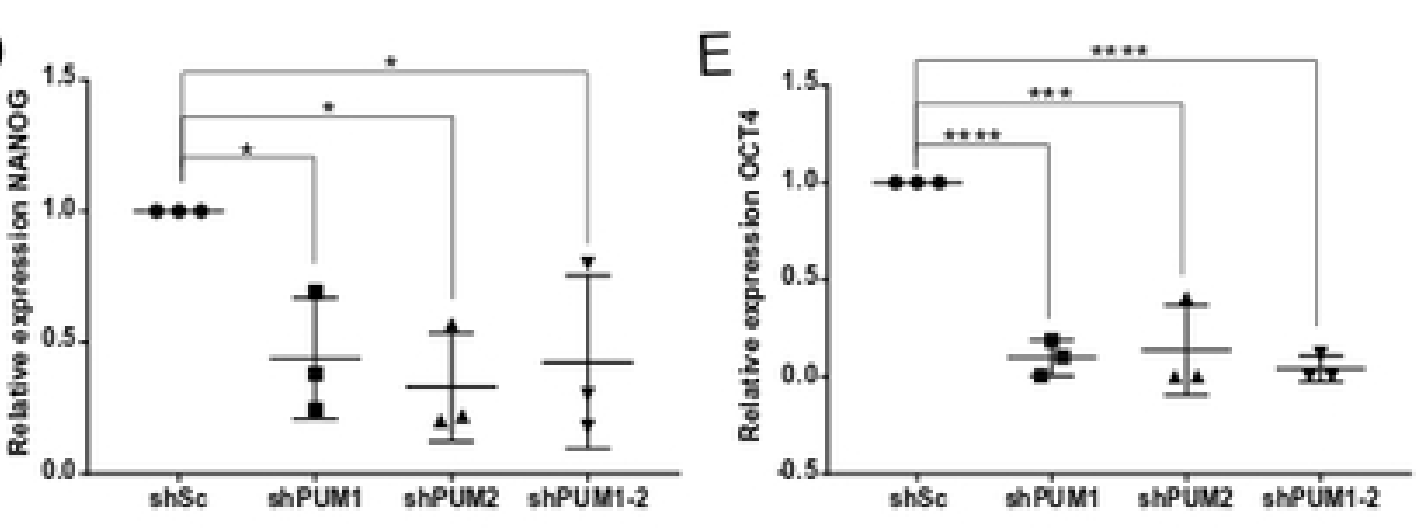

$\mathrm{F}$ shSc

shPUM1-2

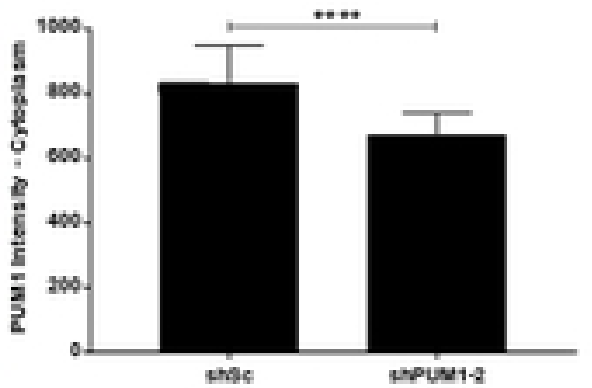

G
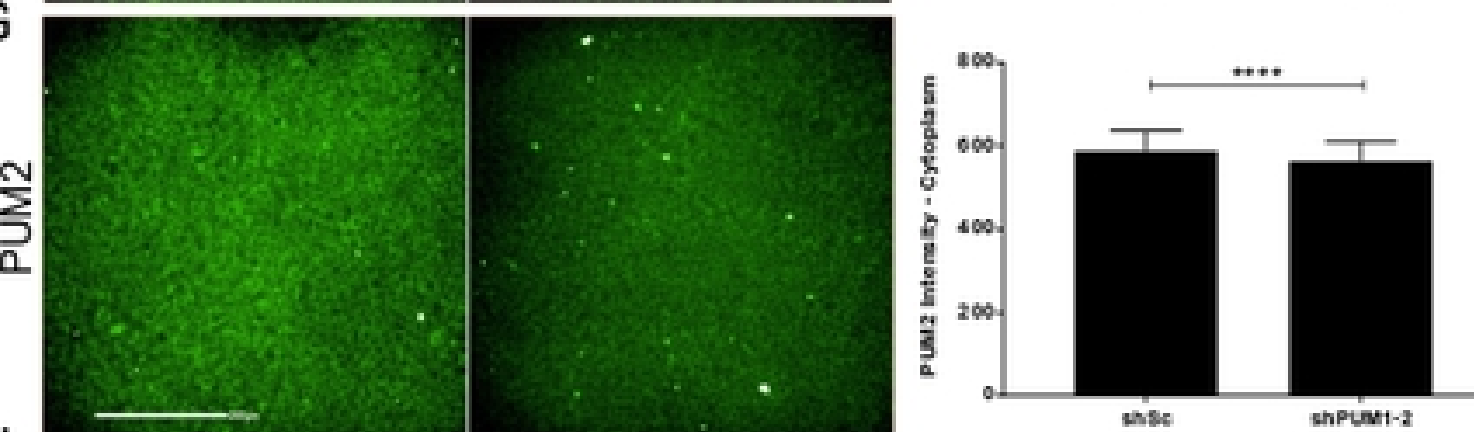

$\mathrm{H}$
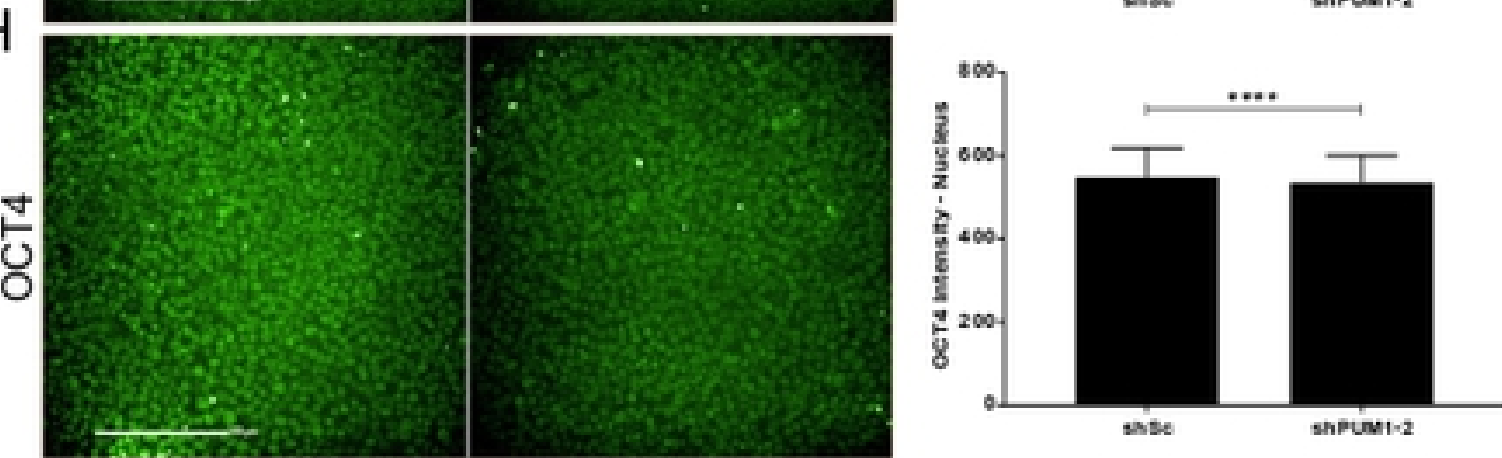

Figure 2 


\section{A}

$\rightarrow$
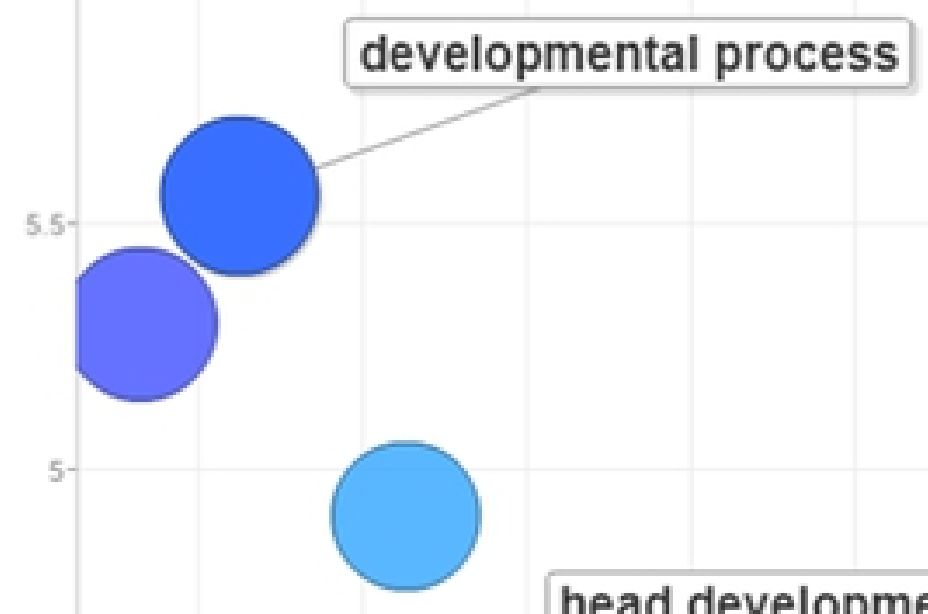

\section{head development}
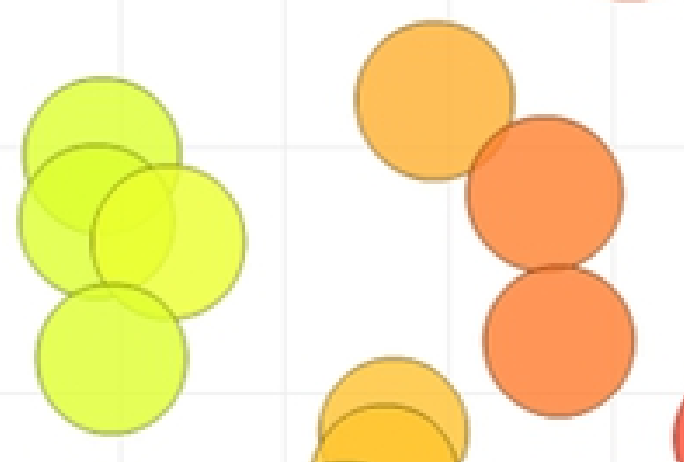

4.5 not certified by peer review) is the author/funder, who has granted bioRxiv a license to display the preprint in perpetuity. It is made

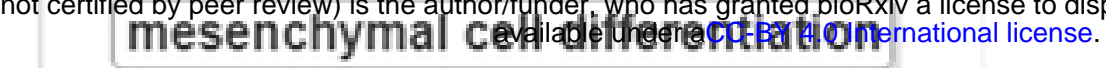

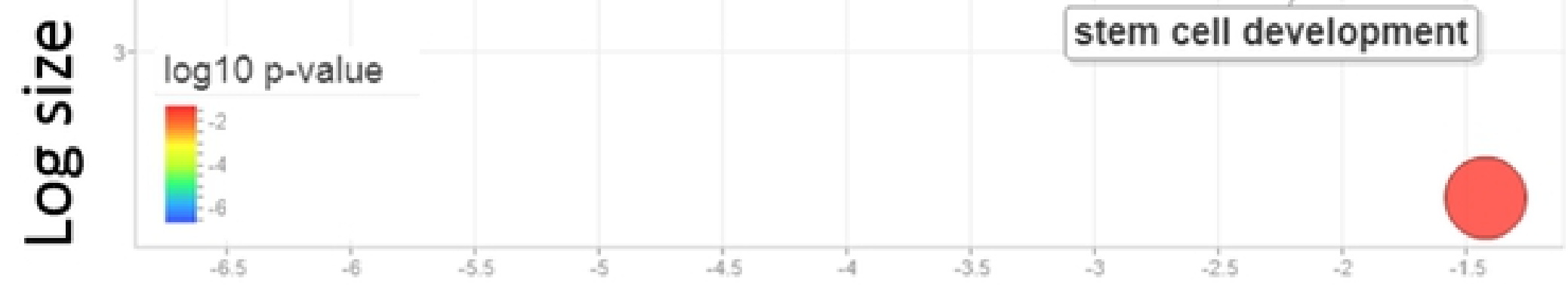

\section{Log10 p-value}

B

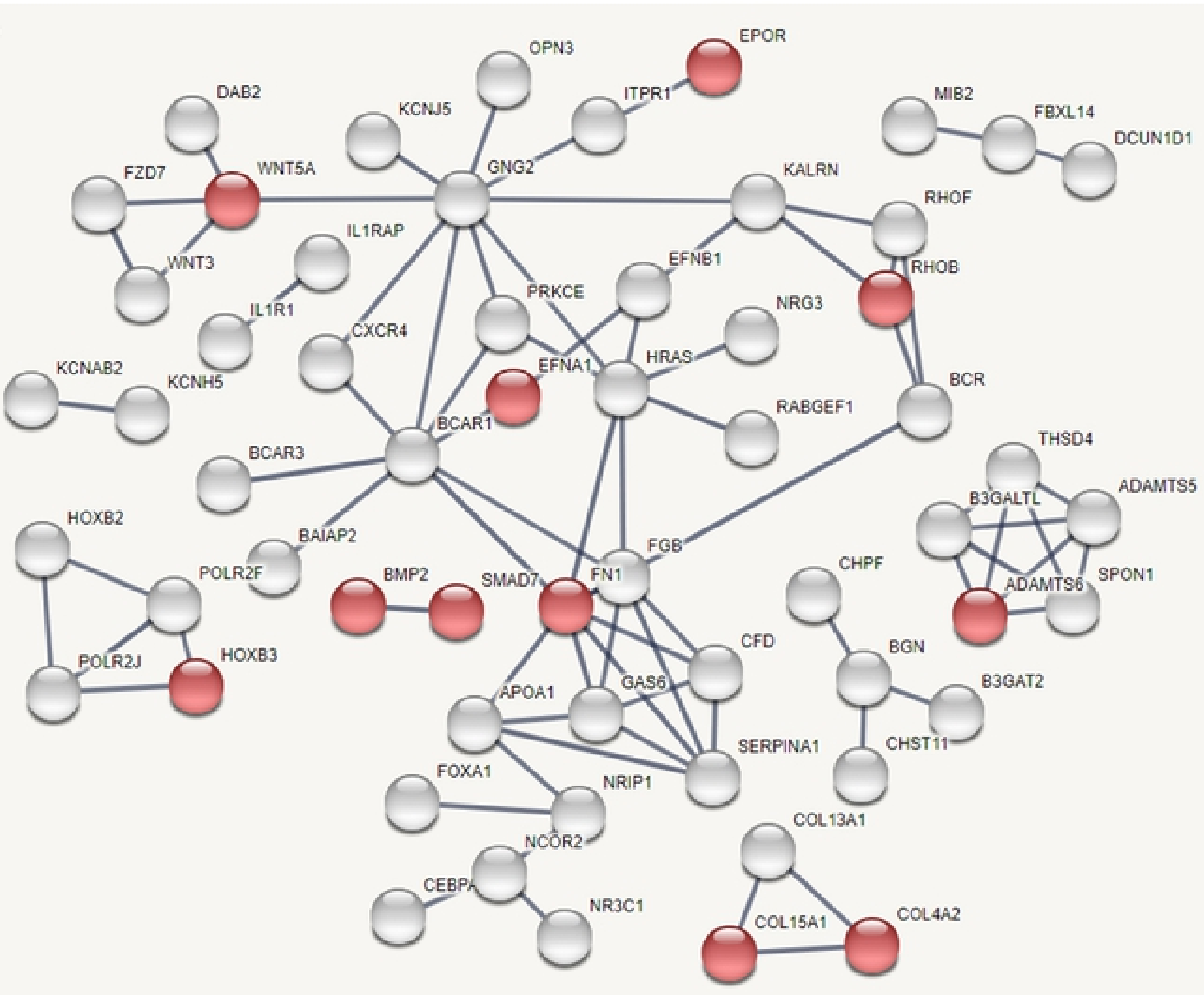

Figure 5 